\title{
Cyclotron-resonance-assisted photocurrents in surface states of a three-dimensional topological insulator based on a strained high-mobility HgTe film
}

\author{
K.-M. Dantscher, ${ }^{1}$ D. A. Kozlov, ${ }^{2,3}$ P. Olbrich, ${ }^{1}$ C. Zoth, ${ }^{1}$ P. Faltermeier, ${ }^{1}$ M. Lindner, ${ }^{1}$ G. V. Budkin, ${ }^{4}$ S. A. Tarasenko, ${ }^{4,5}$ \\ V. V. Bel'kov, ${ }^{4}$ Z. D. Kvon, ${ }^{2,3}$ N. N. Mikhailov, ${ }^{2}$ S. A. Dvoretsky, ${ }^{2}$ D. Weiss, ${ }^{1}$ B. Jenichen, ${ }^{6}$ and S. D. Ganichev ${ }^{1, *}$ \\ ${ }^{1}$ Terahertz Center, University of Regensburg, 93040 Regensburg, Germany \\ ${ }^{2}$ A.V. Rzhanov Institute of Semiconductor Physics, Novosibirsk 630090, Russia \\ ${ }^{3}$ Novosibirsk State University, Novosibirsk 630090, Russia \\ ${ }^{4}$ Ioffe Institute, 194021 St. Petersburg, Russia \\ ${ }^{5}$ St. Petersburg State Polytechnic University, 195251 St. Petersburg, Russia \\ ${ }^{6}$ Paul-Drude-Institut for Solid State Electronics, 10117 Berlin, Germany
}

(Received 23 March 2015; revised manuscript received 5 August 2015; published 27 October 2015)

\begin{abstract}
We report on the observation of cyclotron-resonance-induced photocurrents, excited by continuous wave terahertz radiation, in a three-dimensional topological insulator (TI) based on an 80-nm strained HgTe film. The analysis of the photocurrent formation is supported by complementary measurements of magnetotransport and radiation transmission. We demonstrate that the photocurrent is generated in the topologically protected surface states. Studying the resonance response in a gated sample, we examined the behavior of the photocurrent, which enables us to extract the mobility and the cyclotron mass as a function of the Fermi energy. For high gate voltages, we also detected cyclotron resonance (CR) of bulk carriers, with a mass about two times larger than that obtained for the surface states. The origin of the CR-assisted photocurrent is discussed in terms of asymmetric scattering of TI surface carriers in the momentum space. Furthermore, we show that studying the photocurrent in gated samples provides a sensitive method to probe the cyclotron masses and the mobility of two-dimensional Dirac surface states, when the Fermi level lies in the bulk energy gap or even in the conduction band.
\end{abstract}

DOI: 10.1103/PhysRevB.92.165314

\section{INTRODUCTION}

The physics of relativistic Dirac fermions in semiconductors has recently moved into the focus of modern research, due to their unique properties and promising novel applications (for recent reviews, see [1-6]). Among diverse systems addressed in the literature, HgTe-based structures represent an extraordinary material class, allowing the fabrication of a high-quality material [7-12] with Dirac-type systems of different forms. The latter includes topological protected edge and surface states of two- and three-dimensional topological insulators [13-26], quantum wells (QWs) with critical thickness [13,27-34], and bulk $\mathrm{HgCdTe}$ material at the point of semiconductor-to-semimetal transition [35]. Three-dimensional (3D) topological insulators (TIs) based on strained HgTe films [18] are of particular interest. Indeed, in these materials strain opens a gap in the otherwise gapless $\mathrm{HgTe}$, which together with the high quality of the material allows one to obtain insulation in the bulk and to study electron transport in surface states only [18,24]. This differs significantly from all other known $3 \mathrm{D}$ TIs (e.g., $\mathrm{Bi}_{2} \mathrm{Te}_{3}$, $\mathrm{Sb}_{2} \mathrm{Te}_{3}$ ), where dc electron transport is (almost always) hindered by the high residual carrier density in the bulk [36-38]. This unique property allows one to observe the quantum Hall effect [18] and Shubnikov-de Haas oscillations [24] in strained $\mathrm{HgTe}$ film and, thus, to analyze surface-state transport in TIs. Furthermore, the negligible contribution from bulk carriers opens a way to study Dirac fermions in a 3D TI by cyclotron resonance measured via transmission or Faraday effect [39-41] and, as we show in the following, terahertz $(\mathrm{THz})$ radiation-induced photocurrents.

\footnotetext{
*Sergey.Ganichev@physik.uni-regensburg.de
}

PACS number(s): 73.21.Fg, 72.25.Fe, 78.67.De, 73.63.Hs

Here, we report on the observation of cyclotron-resonanceassisted photocurrents, excited in surface states of strained HgTe films. The analysis of the photocurrent formation in topologically protected surface states is supported by complementary measurements of the radiation transmission and magnetotransport. We demonstrate the photocurrent stems from magnetic field induced asymmetric scattering of nonequilibrium surface carriers in the momentum space. Our analysis of the photocurrent formation suggests that the observed photocurrents are spin polarized and accompanied by a macroscopic surface spin polarization. This fact can be used for studying details of the spin orientation in TI, in particular, to explore the role of bulk and structure inversion asymmetry in the band structure. Moreover, we demonstrate studying the photocurrent provides a sensitive method to probe the cyclotron masses of two-dimensional (2D) Dirac surface states. Importantly, the method is applicable even for micrometer-size gated Hall bar samples for which pure optical methods are almost impossible.

\section{SAMPLES}

The experiments are carried out on molecular beam epitaxy grown high-mobility 80 -nm-thick $\mathrm{HgTe}$ films. The HgTe film is sandwiched between thin $\mathrm{Cd}_{0.65} \mathrm{Hg}_{0.35}$ Te layers acting as capping (top) and buffer (bottom) layers. The cross section of the structure is shown in Fig. 1(a). The structure is grown on a GaAs substrate with (013) surface orientation covered by a thin ZnTe layer and $4-\mu$ m-thick CdTe layer. The CdTe layer is fully relaxed because of its large thickness. The Cd$\mathrm{HgTe} / \mathrm{HgTe} / \mathrm{CdHgTe}$ layers grown on top adopt the underlying $\mathrm{CdTe}$ lattice constant. The lattice mismatch between $\mathrm{HgTe}$ and CdTe of about $0.3 \%$ results in a tensile strain in the $\mathrm{HgTe}$ film, which opens a topological gap $[15,16,18]$. The evidence for 


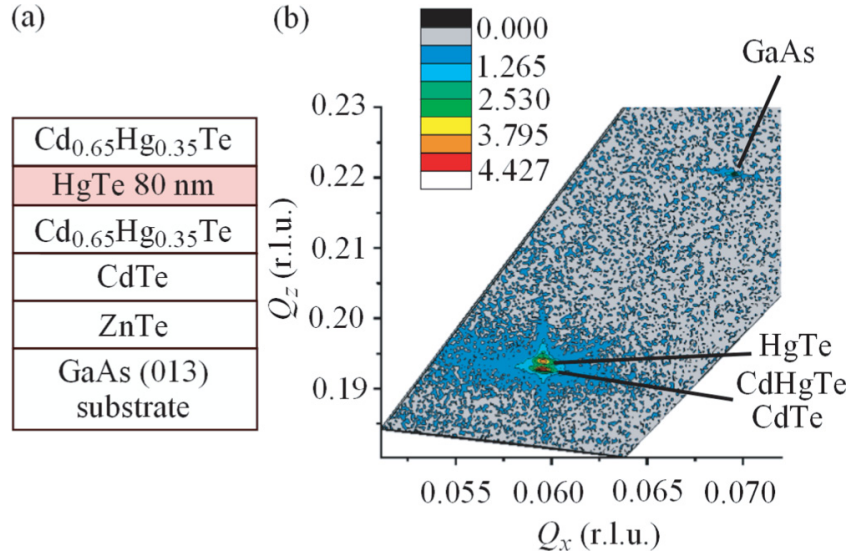

FIG. 1. (Color online) (a) Cross section of the investigated structures. (b) An X-ray reciprocal space map near the asymmetric (113) reflection. The diffracted intensity is plotted on a color-coded logarithmic scale. The CdTe film is relaxed with respect to the GaAs substrate because the line connecting their maxima points to the origin of the reciprocal space. The $\mathrm{HgTe}$ and $\mathrm{CdHgTe}$ films are fully strained with respect to the $\mathrm{CdTe}$, as their peaks lie on a straight line along the $z$ axis perpendicular to the sample surface (r. l. u.:- reciprocal lattice unit).

substantial strain in our samples comes from x-ray diffraction measurements, shown in Fig. 1(b). Note that such a sandwich design of the structure reduces the influence of dislocations caused by the lattice mismatch [24] and allows one to obtain high electron mobilities (up to $4 \times 10^{5} \mathrm{~cm}^{2} / \mathrm{V} \mathrm{s}$ at $40 \mathrm{~K}$ ) together with low residual bulk impurity concentration (about $10^{16} \mathrm{~cm}^{-3}$ as estimated for ungated samples).

Several kinds of gated and ungated samples have been prepared from the same wafer, including ungated squareshaped samples of $4 \times 5 \mathrm{~mm}$ size [see Fig. 2(a)], gated (ungated) cross-shaped structures [see Fig. 2(b)], and gated Hall-bar samples. On the square-shaped samples we have fabricated four Ohmic contacts in the middle of the edges. The sample edges are oriented along $x \|[100]$ and $y \|[03 \overline{1}]$. The samples of this kind have been used for simultaneous transmission and photocurrent measurements. In order to study photocurrents and magnetotransport as a function of the Fermi level position, we have fabricated gated structures. To avoid insulator leakages, we have used small area cross-shaped structures with four Ohmic contacts [Fig. 2(b)]. The structures of $50-\mu \mathrm{m}$ width and $1500-\mu \mathrm{m}$ length have been patterned by means of standard photolithography and wet etching. Semitransparent Ti/Au gates of $20-\mathrm{nm} / 5-\mathrm{nm}$ thickness and
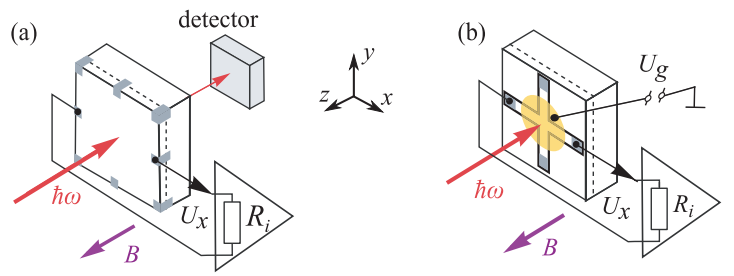

FIG. 2. (Color online) Experimental setups of (a) the photocurrent and radiation transmission measurements in squared-shaped samples and (b) photocurrent measurements in cross-shaped samples.
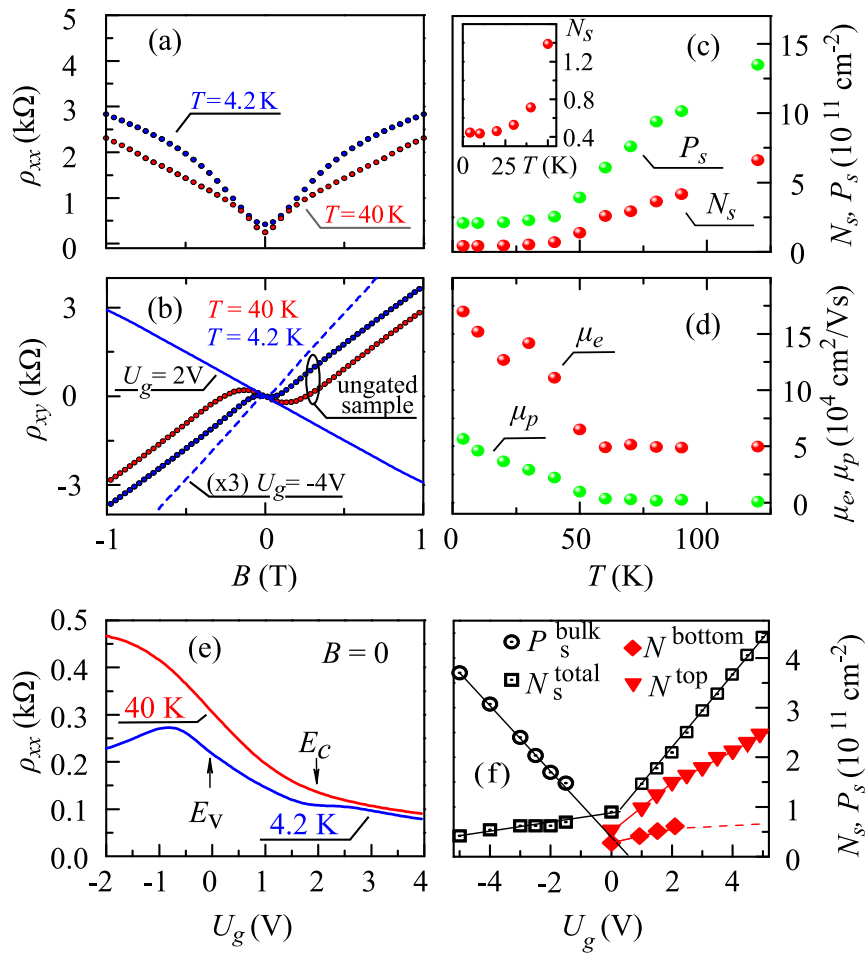

FIG. 3. (Color online) Magnetotransport data obtained in ungated cross-shaped [(a)-(d)], gated cross-shaped [(b) and (e)], and gated Hall-bar (f) samples. The panel (a) shows the $\rho_{x x}$ and (b) the $\rho_{x y} B$ dependence for $T=4.2$ and $40 \mathrm{~K}$. (c) Electron $N_{s}$ and hole $P_{s}$ densities and (d) mobilities $\left(\mu_{e}\right.$ and $\left.\mu_{p}\right)$ at different temperatures, extracted from the Drude model. (e) $U_{g}$ dependence of $\rho_{x x}$ at $T=4.2$ and $40 \mathrm{~K}$. The valence and conduction band edges are marked by arrows " $E_{v}$ " and " $E_{c}$," respectively. (f) Electron and hole densities of bulk ( $N_{s}^{\text {total }}$ and $\left.P_{s}^{\text {bulk }}\right)$ and surface states $\left(N^{\text {top }}\right.$ and $\left.N^{\text {bottom }}\right)$. The data are obtained at $T=1.9 \mathrm{~K}$ on the same sample as that used in Ref. [24] but at a different cooldown. While the overall characteristic of the data is the same, we note that the position of the gap is shifted on the gate voltage scale. This is ascribed to cooldown-dependent charge trapping in the insulator. For the photocurrent experiments described below we made sure that the transport experiments were done under the same conditions as the cyclotron resonance experiments, i.e., had the charge neutrality point fixed and has the same value for all investigated samples.

1500- $\mu \mathrm{m}$ diameter have been deposited on $100 \mathrm{~nm} \mathrm{SiO}_{2}$ and $200 \mathrm{~nm}$ of $\mathrm{Si}_{3} \mathrm{~N}_{4}$ dielectric layer grown by chemical vapor deposition. To monitor the influence of the gates on the CR position, we have used both gated as well as ungated cross structures. Gates have also been deposited on Hall-bar samples, which have been used primarily for magnetotransport experiments.

Samples have been characterized by magnetotransport measurements using standard low-frequency lock-in technique with the currents in the range of $0.1-1 \mu \mathrm{A}$ and with the magnetic field $B$ up to $7 \mathrm{~T}$ applied normal to the $\mathrm{HgTe}$ film plane. The measurements of $\rho_{x x}(B)$ and $\rho_{x y}(B)$ for the ungated sample at $T=4.2$ and $40 \mathrm{~K}$ are presented in Figs. 3(a) and 3(b). The obtained $\rho_{x x}(B)$ shows a large positive magnetoresistance and the Hall resistance $\rho_{x y}(B)$ exhibits a nonlinear $N$-type shape around $B=0 \mathrm{~T}$, both of which are typical for a 
system with coexisting electrons and holes. The corresponding quantized Hall data are shown in Ref. [24]. Fitting these traces by using the classical two-component Drude model we obtain electron and hole densities and mobilities [24]. The results of the fitting are presented in Figs. 3(c) and 3(d). The temperature dependence of electron and hole densities shows typical activation behavior at $T>50 \mathrm{~K}$. The presence of holes at low temperatures indicates that the Fermi level is situated in the valence band while the observed electrons are attributed to the surface topological states.

To support this conclusion, we studied magnetotransport in gated samples. By means of a top gate, the Fermi energy was tuned from the valence band through the Dirac-type surface states into the conduction band. The $\rho_{x x}\left(U_{g}\right)$ dependence obtained at $T=4.2$ and $40 \mathrm{~K}$ is presented in Fig. 3(e), showing that the low-temperature resistivity has a maximum near $U_{g} \approx-0.8 \mathrm{~V}$. At the same gate voltage, the Hall resistivity $\rho_{x y}$ changes its sign at $B=1 \mathrm{~T}$ [see Fig. 3(b)]. The analysis of the data, carried out following Ref. [24], delivers a selfconsistent picture of the gate-controlled transition between a hole metal to a topological insulator and, finally, at large positive gate voltages, to an electron metal. The edges of the bulk bands are well defined; see arrows marked " $E_{v}$ " and " $E_{c}$ " in Fig. 3(e) for valence and conduction band edges, respectively. In the whole range of $U_{g}$, corresponding to the Fermi level situated in the valence band, one observes a large positive magnetoresistance accompanied by a nonlinear (N-shaped) $\rho_{x y}(B)$, which changes sign. This indicates that both electrons and holes contribute to transport and the results can be nicely fitted by a two-carrier Drude model (see Ref. [24]). The electron and hole 2D densities of bulk and surface states as a function of $U_{g}$ are shown in Fig. 3(f). The same transport signatures have been observed for the ungated samples [see Figs. 3(a) and 3(b)], indicating that the Fermi level is situated in the valence band as well. When the Fermi level is in the valence band, the conduction band is about $15 \mathrm{meV}$ (energy gap) above so that at 4.2 or $40 \mathrm{~K}$ the thermal occupation of the conduction band states is small. Therefore, only surface electrons contribute for these gate voltages and temperatures to transport. Note that at high temperatures electrons populate surface as well as bulk states. Figure 3(d) shows that both, electron and hole, mobilities decrease with increasing temperature. We emphasize that the electron mobility remains as high as $10^{5} \mathrm{~cm}^{2} / \mathrm{V} \mathrm{s}$ even at $T=40 \mathrm{~K}$.

This indicates that gate fabrication does not change the band diagram of the structure qualitatively but only introduces a built-in electric field due to, e.g., charged impurities and/or defects. The defect density can be estimated as $(1 \div 2) \times$ $10^{11} \mathrm{~cm}^{-2}$, varying from sample to sample.

For photocurrent excitation and cyclotron-resonance transmission measurement we apply a cw molecular laser $[42,43]$ emitting radiation with frequency $f=2.54 \mathrm{THz}$ (wavelength $\lambda=118 \mu \mathrm{m}), f=1.62 \mathrm{THz}(\lambda=184 \mu \mathrm{m})$, and $f=$ $0.69 \mathrm{THz}(\lambda=432 \mu \mathrm{m})$. The incident power $P \approx 10 \mathrm{~mW}$ is modulated at about $180 \mathrm{~Hz}$ by an optical chopper. Structures were placed in a temperature variable optical cryostat with $z$-cut crystal quartz windows. The radiation at normal incidence is focused onto a spot of about $1.5-\mathrm{mm}$ diameter at the center of the sample. The spatial beam distribution has
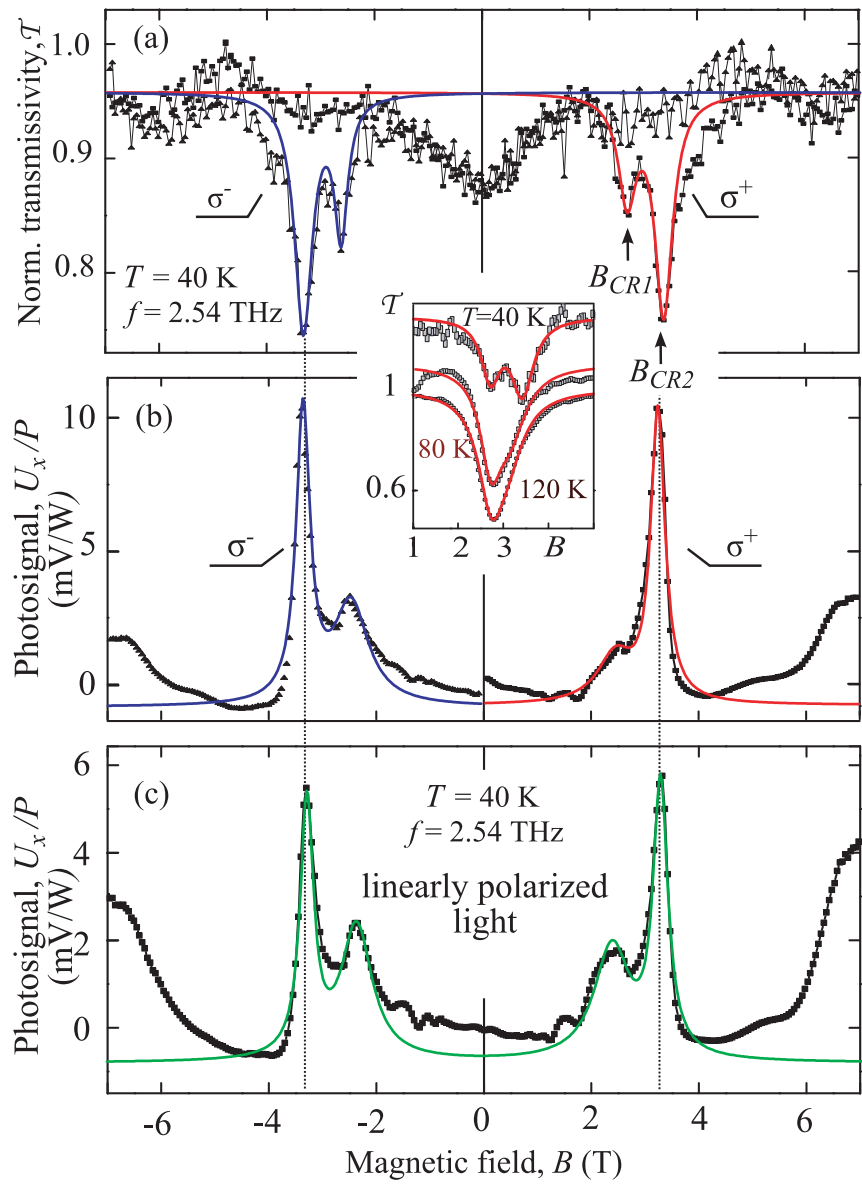

FIG. 4. (Color online) (a) Normalized transmissivity of the square-shaped $\mathrm{HgTe}$ sample at $40 \mathrm{~K}$ as a function of magnetic field. The data are given for right $\left(\sigma^{+}\right)$and left $\left(\sigma^{-}\right)$circularly polarized light. The inset shows the averaged transmissivity traces obtained for different temperatures. (b), (c) Show the photosignal normalized by the radiation power $\left(U_{x} / P\right)$ for $\sigma^{+}$and $\sigma^{-}$and for linearly polarized radiation, respectively. Full lines in all panels show fits by the Lorentzian function.

an almost Gaussian profile, measured by a pyroelectric camera $[44,45]$. The initial linear polarization can be transformed into right- $\left(\sigma^{+}\right)$and left- $\left(\sigma^{-}\right)$handed circularly polarized light by applying a $\lambda / 4$ plate. A magnetic field $B$ up to $7 \mathrm{~T}$ is applied normal to the film. The photoresponse is measured applying standard lock-in technique, either as a photocurrent $j_{x, y}$ or the corresponding photovoltage $U_{x, y}$ picked up across $50 \Omega$ or $10 \mathrm{M} \Omega$ load resistors, respectively. Parallel to the photocurrents we also studied radiation transmission [see Fig. 4(a)]. For that a Golay cell detector has been placed behind the sample.

\section{EXPERIMENTAL RESULTS}

We begin with the transmissivity data obtained from the square-shaped sample. The data for $T=40 \mathrm{~K}$ are shown in Fig. 4(a) for different polarization states. Exciting the sample with right-handed circularly polarized radiation $\left(\sigma^{+}\right)$ and sweeping the magnetic field, we observe two resonant dips at positive magnetic fields $B=2.6$ and $3.35 \mathrm{~T}$. Upon changing the radiation helicity from $\sigma^{+}$to $\sigma^{-}$, the dips appear 
at negative magnetic fields. For linearly polarized radiation, the resonances are observed for both magnetic field directions (not shown). The resonances can be well fitted by a Lorentzian function. All these features are clear signs that the absorption of radiation is caused by cyclotron resonance of electrons. The dips in the transmissivity $\mathcal{T}$ are detected in the temperature range from 4.2 up to $150 \mathrm{~K}$. Below $4.2 \mathrm{~K}$, the amplitude of both dips is smaller than the noise level of our setup. With rising temperature, the two dips increase in amplitude and merge together at $T$ above $\sim 80 \mathrm{~K}$ [see inset of Fig. 4(a)]. The temperature dependence is due to the increasing carrier density with higher temperature and has been independently checked by magnetotransport data on the same sample [see Fig. 3(c)]. These results are in agreement with findings of Refs. [40,41] where double CR structures have been detected in similar systems by transmission and Faraday effect applying radiation of lower frequencies and attributed to top and bottom surface states.

From the cyclotron-resonance positions given by

$$
B_{\mathrm{CR}}=2 \pi f \frac{m_{\mathrm{CR}}}{|e|},
$$

where $e$ is the electron charge, we determined the correspondent cyclotron masses $m_{\mathrm{CR}}$. At $T=40 \mathrm{~K}$ we obtained the masses $m_{\mathrm{CR}}=0.028 m_{0}$ and $0.035 m_{0}$, for dips at $B=2.6$ and $3.35 \mathrm{~T}$, respectively.

The masses agree well with the estimated cyclotron masses of surface states. We carried out $\boldsymbol{k} \cdot \boldsymbol{p}$ calculations of the band structure for the (013)-oriented 80-nm-wide $\mathrm{HgTe}$ film sandwiched between $\mathrm{CdHgTe}$ barriers adapting the procedure described in the Supplemental Material to Refs. [18] and [29,46]. We calculated the energy spectrum in zero magnetic field and then analyzed the Bohr-Sommerfeld/quasiclassical quantization in a magnetic field which allowed us to estimate the cyclotron mass. The details of our calculations for (013)oriented structures taking into account built-in electric fields are given in the Appendix.

The obtained band structure and cyclotron masses as a function of the energy are shown in Fig. 5(a) and the inset, respectively. The calculations reveal the presence of surface states in the band gap of bulk strained HgTe. The Dirac points of the surface states for both top and bottom interfaces are deep in the valence band, which leads to level mixing and deviation of the energy dispersion from the linear one, similarly to the results obtained for (001)-oriented structures [18]. In (013)grown structures, the energy spectrum is anisotropic in the interface plane. To illustrate the anisotropy, we plotted the band structure in Fig. 5(a) calculated for two perpendicular in-plane directions (solid and dashed lines). Moreover, compared to (001)-oriented films, we find that the spin carried by the surface electrons is tilted out of the interface plane, reflecting the low spatial symmetry of the (013)-oriented structure (see Appendix). Our calculation shows that the masses for surface states are very close to the experimentally detected ones and, also in agreement with experiment, vary only weakly with temperature [see Fig. 5(b)]. The detail comparison between the experimentally observed cyclotron masses and the calculated values require the knowledge of electric field distribution and chemical composition profile in real structures and is out of scope of this paper. We note that the density of
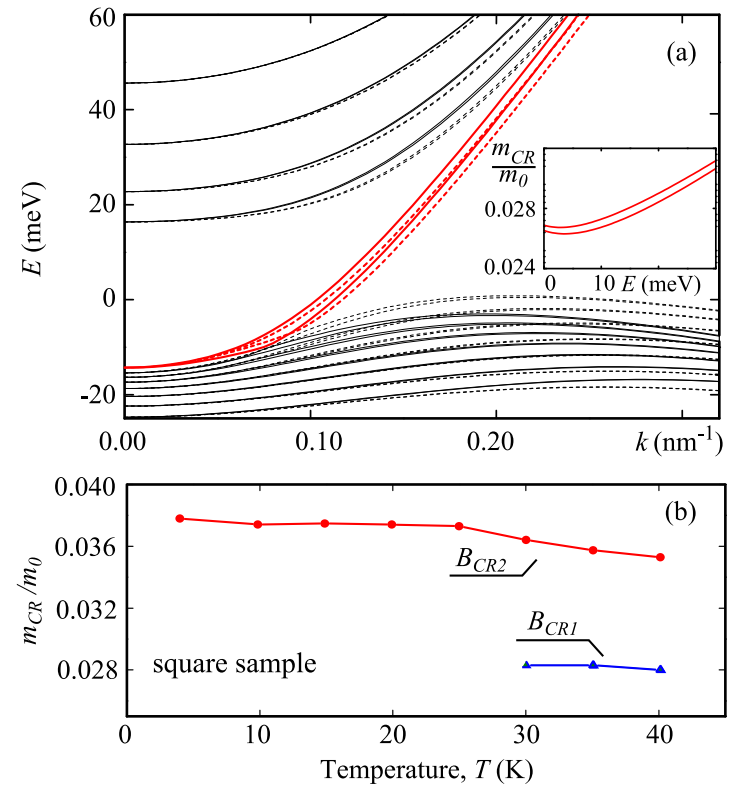

FIG. 5. (Color online) (a) The band structure of (013)-grown 80 -nm strained $\mathrm{HgTe}$ film calculated by $\boldsymbol{k} \cdot \boldsymbol{p}$ model. Solid and dashed lines show the band dispersion for two perpendicular in-plane crystallographic directions: $\boldsymbol{k} \|$ [100] (solid curves) and $\boldsymbol{k} \|[03 \overline{1}]$ (dashed curves). Calculations are carried out for built-in electric field $E_{z}=0.5 \mathrm{kV} / \mathrm{cm}$ which results in the energy splitting of the top and bottom surface states, shown by thick red solid and dashed curves. Inset shows calculated cyclotron masses for surface states as a function of energy. (b) Cyclotron masses obtained from the transmission measurements for $\left(\sigma^{+}\right)$light and $f=2.54 \mathrm{THz}$. Dots and triangles show the results for two CR lines.

bulk states in the valence band is very high due to large effective mass of holes. Therefore, the Fermi level in ungated samples and in gated samples at negative voltages is efficiently pinned to the valence band top. Thus, we conclude that the resonances are caused by the top/bottom surface states. This conclusion is also supported by magnetotransport data indicating a vanishing amount of bulk electrons. Moreover, according to the calculation, cyclotron resonance of the bulk carriers is expected at substantially higher magnetic fields corresponding to a mass of about $0.07 m_{0}$.

Next, we demonstrate that the CR, so far observed in transmission, results in a photocurrent, whose magnetic field and polarization dependencies fully reproduce the peak positions and shapes of the $\mathrm{CR}$ absorption. The magnetic field dependencies of the corresponding photosignals are shown for circularly or linearly polarized radiation in Figs. 4(b) and 4(c), respectively. The coincidence of the transmission and photocurrent resonance positions indicates that the current is also excited in the surface states. For a more detailed study of the photocurrent we used cross-shaped samples, which are too small to be used for transmission measurements, but allow fabrication of semitransparent gates.

Figure 6 shows the resonant photocurrent obtained for different radiation frequencies in an ungated cross-shaped sample. Comparison of the data for $f=2.54 \mathrm{THz}$ obtained for square- and crossed-shaped samples shows that magnetic field positions of the resonances stay almost the same. Notice 


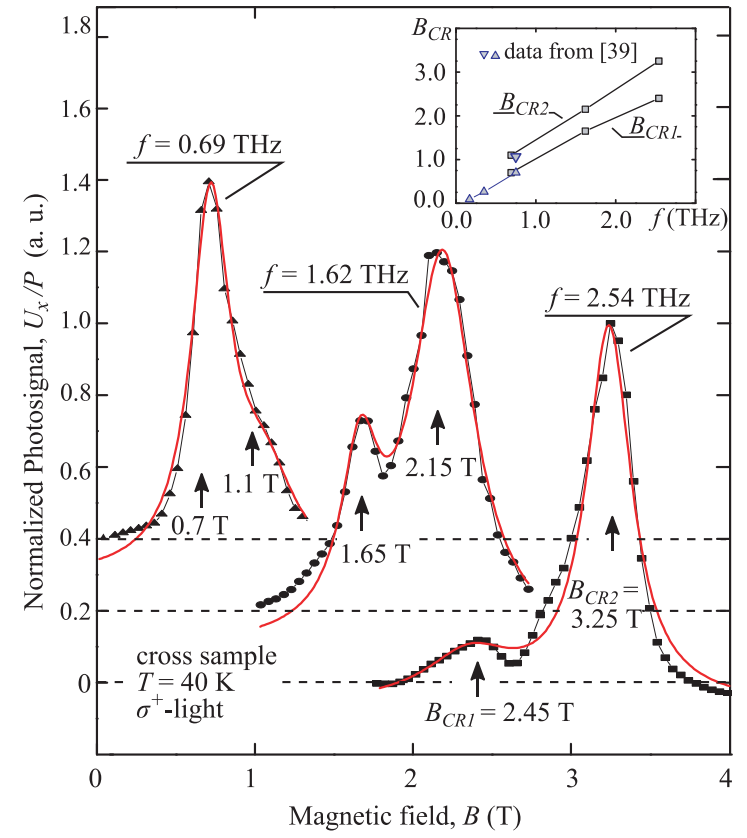

FIG. 6. (Color online) Normalized CR radiation-induced photocurrent measured for different radiation frequencies in ungated cross-shaped samples. Note that the data are $y$ shifted by 0.2 for and 0.4 for $f=1.62$ and $0.69 \mathrm{THz}$, respectively. Full lines show fits by the Lorentzian function. Inset shows the first $B_{\mathrm{CR} 1}$ (squares) and second $B_{\mathrm{CR} 2}$ (circles) resonance positions as a function of frequency. Full and open triangles show the data of Ref. [39] for top and bottom surface states measured in similar strained $\mathrm{HgTe}$ film of $70 \mathrm{~nm}$ width.

that the relative strength of the first $B_{\mathrm{CR} 1}$ and second $B_{\mathrm{CR} 2}$ resonances substantially change with decreasing frequency. While at $f=2.54 \mathrm{THz}$ the second resonance is substantially stronger than the first one, for $f=0.69 \mathrm{THz}$ the situation reverses. The inset in Fig. 6 demonstrates that the position of both resonances linearly scales with the frequency. The frequency dependence of the CR is in line with transmissivity data of Ref. [39] of top and bottom surface states, obtained for a strained HgTe films of $70 \mathrm{~nm}$ width (see triangles in the inset in Fig. 6).

In order to provide additional support for the conclusion that the observed resonant photocurrent and CR stem from twodimensional surface states, we carried out measurements with the magnetic field tilted by an angle $\Theta$. Figure 7 shows that the current is caused by the magnetic field component $B_{z}$, normal to the surface. Indeed, calculating from the peak position $B_{\mathrm{CR}}$ in Fig. 7, the value of $B_{z}^{\mathrm{CR}}=B_{\mathrm{CR}} * \cos (\Theta)$, we obtain that $B_{z}^{\mathrm{CR}}$ does not depend on the angle $\Theta$ (see inset). Moreover, for an in-plane magnetic field the photocurrent vanishes (not shown), indicating the two-dimensional nature of the carriers [47].

By using gated cross-shaped samples we study the photocurrent and $\mathrm{CR}$ as a function of the Fermi energy [see Figs. 8(a) and 8(b)]. The data show that at negative-bias voltages, for which the Fermi energy lies in the valence band, the photocurrent resonances vanish. At positive gate voltages, the resonances in photocurrent at $B_{\mathrm{CR} 2}=3.3 \mathrm{~T}$ and $B_{\mathrm{CR} 1}$ at lower magnetic field are clearly resolved at both $T=40$ and $20 \mathrm{~K}$. The positions of the resonances are similar to those obtained for ungated samples (see Figs. 4 and 6), and

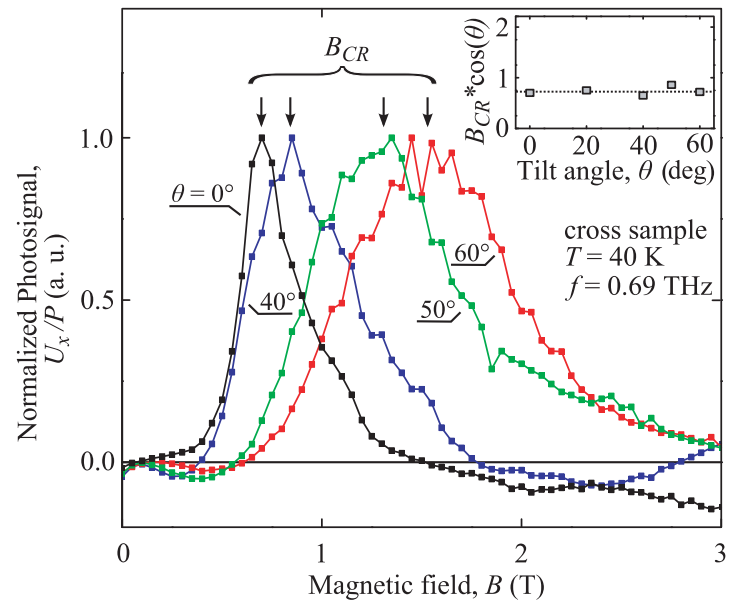

FIG. 7. (Color online) (a) Magnetic field dependencies of the photocurrent measured for magnetic field tilted by an angle $\Theta$ and linearly polarized light. The inset shows the resonance field $B_{z}^{\mathrm{CR}}=B_{\mathrm{CR}} * \cos (\Theta)$ as a function of angle $\Theta$.
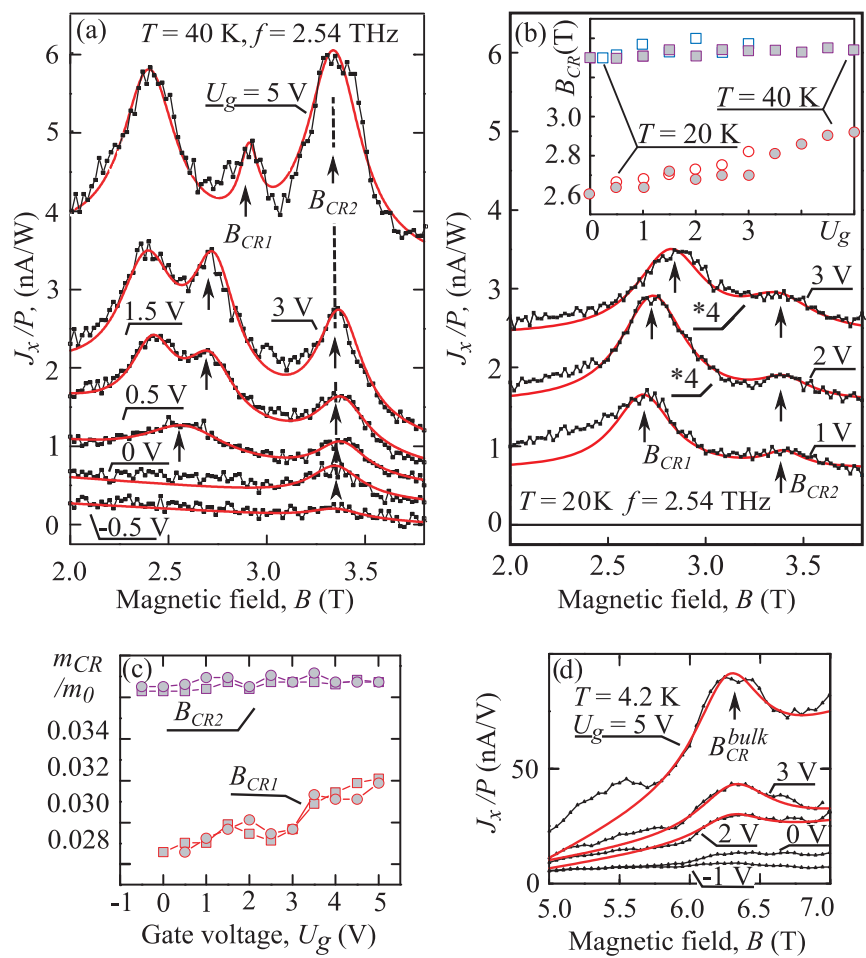

FIG. 8. (Color online) (a), (b) Magnetic field dependence of the photocurrent measured for the gated cross sample \#1 at different gate voltages $U_{g}$ at $T=40$ and $20 \mathrm{~K}$, respectively. Full lines show fits using a Lorentzian. Note that the data for various $U_{g}$ are $y$ shifted for clarity. In panel (b) the data for $U_{g}=2$ and $3 \mathrm{~V}$ are multiplied by a factor 4 . The inset in panel (b) shows $B_{\mathrm{CR} 1,2}$ determined from $J_{x}(B)$ for both temperatures. (c) Gate voltage dependencies of cyclotron masses obtained from the position of CR resonances $B_{\mathrm{CR} 1,2}$ determined from $J_{x}(B)$ and $J_{y}(B)$. (d) Magnetic field dependence of the photocurrent measured at low temperature and high magnetic fields. 
correspond to the response of the top and the bottom surface states of the film. At $U_{g}>1 \mathrm{~V}$ and $T=40 \mathrm{~K}$, we detect an additional resonance at $B \approx 2.4 \mathrm{~T}$, which only appears at $40 \mathrm{~K}$ but is absent at $20 \mathrm{~K}$. The origin of this extra structure, which was observed in some samples at elevated temperatures is at present not understood and requires further experiments. At even higher gate voltages $U_{g}>2 \mathrm{~V}$ and $T=4.2 \mathrm{~K}$, we also detected another resonance at much stronger magnetic fields $B \approx 6.3 \mathrm{~T}$ [see Fig. 8(d)]. The position of this resonance does not depend on the gate voltage and the corresponding cyclotron mass is $m_{\mathrm{CR}}=0.07 m_{0}$. This mass matches the one calculated for bulk electrons, so that the resonance can be attributed to the $\mathrm{CR}$ of bulk electrons. We note that at $T=4.2 \mathrm{~K}$, the surface-state resonances are superimposed by Shubnikov-de Haas oscillations, which makes the CR analysis almost impossible.

From the resonance positions of the surface-state response, we determine the gate voltage dependence of the cyclotron masses [see Fig. 8(c)]. While the mass corresponding to $B_{\mathrm{CR} 2}$ remains almost independent of the gate voltage, the $B_{\mathrm{CR} 1}$ slightly rises with increasing $U_{g}$. The resonance at $B_{c} \approx 3.3 \mathrm{~T}$ $\left(m_{\mathrm{CR}} \approx 0.036 m_{0}\right)$ is attributed to the bottom surface, whose electron density is not much influenced by the gate voltage. In fact, it is known from the magnetotransport measurements [see Fig. 3(f)] that the filling rates $d N_{s} / d U_{g}$ for top and bottom surface states are considerably different because of electrostatic screening of the bottom surface by the top one. Moreover, the electron density at the top surface rises with the gate voltage increase [see Fig. 3(f)], which leads to the increase of the cyclotron mass. By contrast, the density of the bottom electrons is almost independent of the gate voltages [see Fig. 3(f)].

The theory which will be described following shows that the magnitude of surface photocurrents is determined by many parameters, including the carrier density and the asymmetry of electron scattering in momentum space, which all may depend on the gate voltage. Therefore, a connection between the photocurrent magnitude and the carrier density is intricate and the signal height does not allow drawing conclusions regarding the carrier density. For example, while at $T=40 \mathrm{~K}$ the photosignal amplitude at $B_{\mathrm{CR} 2}$ increases with the gate voltage, it decreases at lower temperature $T=20 \mathrm{~K}$ [see Figs. 8(a) and 8(b)]. This difference may stem from the influence of the gate voltage on the asymmetry of electron scattering in the momentum space which underlies the photocurrent formation.

From the full widths at half maximum of the CR measured in transmission in square-shaped ungated samples [see Fig. 4(a)], we also estimate the mobility of the surface electrons, being $\mu_{e}=5.5 \times 10^{4} \mathrm{~cm}^{2} / \mathrm{V} \mathrm{s}$. This value fits well to the mobility obtained from magnetotransport in ungated cross-shaped samples [see Fig. 3(d)]. Finally, we discuss the photocurrent behavior when reversing the magnetic field direction. The magnetic field dependence of the photocurrent measured for two in-plane directions ( $x$ and $y$ ) is shown in Fig. 9. First of all, this plot indicates that both resonances behave independently of each other. Indeed, while the resonance for the photosignal $U_{x}$ at $B_{\mathrm{CR} 1}$ is even in magnetic field, the one at $B_{\mathrm{CR} 2}$ is odd (see inset in Fig. 9 presenting the sum and the difference for negative and positive magnetic fields [48]). This result agrees well with the above conclusion that the

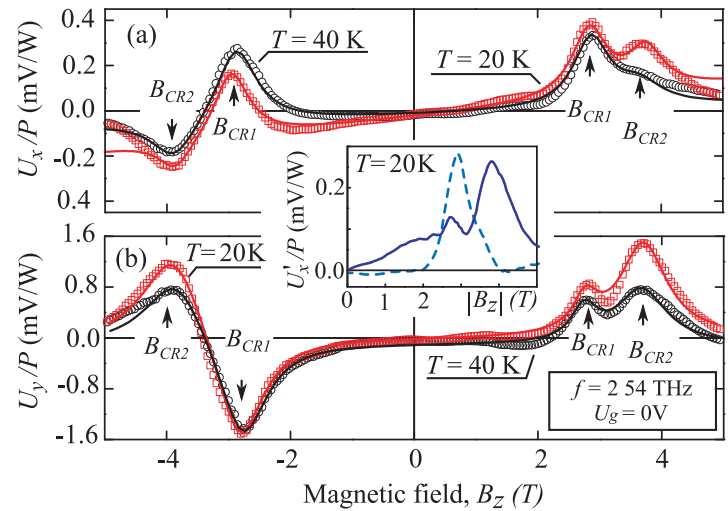

FIG. 9. (Color online) Magnetic field dependence of the photosignal measured for two in-plane directions $(x$ and $y)$ obtained for gated cross sample \#2 $\left(U_{g}=0\right)$. The inset presents the normalized signal $U_{x}^{\prime}$ calculated as one-half of the sum (dashed line) and the difference (solid line) for signals measured at negative and positive magnetic fields $U_{x}^{\prime}=\left[U_{x}(B>0) \pm U_{x}(B<0)\right] / 2$.

photocurrents are generated by two independent surface states (top and bottom). For the other direction, this situation just reverses: Now, the resonance at $B_{\mathrm{CR} 1}$ is odd and $B_{\mathrm{CR} 2}$ is even in magnetic field.

Our findings can be well understood in the framework of the asymmetric photoexcitation and/or energy relaxation of a nonequilibrium electron gas. The appearance of magnetic field induced photocurrents, excited by homogeneous radiation, is well known for two-dimensional systems lacking spatial inversion symmetry (for review, see Refs. [49,50]).

For free carrier absorption, being accompanied by electron scattering from phonons or static defects, the current is caused by the asymmetry of electron scattering in momentum space induced by an external magnetic field, when the electron systems are driven out of thermal equilibrium by light absorption. In the presence of a magnetic field, the rate of the scattering between the states with the momenta $\boldsymbol{p}$ and $\boldsymbol{p}^{\prime}$ contains asymmetric $\boldsymbol{B}$-field-dependent terms (odd in the electron wave vector) and can be presented in the form

$$
W_{\boldsymbol{p}^{\prime} \boldsymbol{p}}=W_{\boldsymbol{p}^{\prime} \boldsymbol{p}}^{(0)}+W_{\boldsymbol{p}^{\prime} \boldsymbol{p}}^{(1)} B
$$

where $W_{\boldsymbol{p}^{\prime} \boldsymbol{p}}^{(0)}$ is the scattering rate at zero field and $W_{\boldsymbol{p}^{\prime} \boldsymbol{p}}^{(1)}$ is an asymmetric correction caused by the mixing of electron states by the magnetic field [51]. The terms in Eq. (2) satisfy $W_{\boldsymbol{p}^{\prime} \boldsymbol{p}}^{(0)}=W_{-\boldsymbol{p}^{\prime},-\boldsymbol{p}}^{(0)}$ and $W_{\boldsymbol{p}^{\prime} \boldsymbol{p}}^{(1)}=-W_{-\boldsymbol{p}^{\prime},-\boldsymbol{p}}^{(1)}$ due to time inversion symmetry.

The asymmetry of the carrier scattering in momentum space, which is caused by different scattering rates to the states with oppositely oriented momenta, leads to electric current generation. We note that at topological surface states, the electrons spin orientation is locked to its momentum [4,5]. This suggests that the observed photocurrents are spin polarized and accompanied by the emergence of a macroscopic surface spin polarization. As a specific feature of (013)-oriented surface, this spin polarization has a nonvanishing out-ofplane component (see Appendix). At cyclotron resonance, the electron-photon interaction is enhanced, which results in a resonant behavior of the photocurrent. 
Considering the generation of an asymmetric electron distribution in momentum space by $\mathrm{cw}$ radiation, the distribution evolution in the magnetic field and its decay due to scattering processes, one can write the Boltzmann equation for the distribution function $f_{p}$ :

$$
e[\boldsymbol{v} \times \boldsymbol{B}] \cdot \frac{\partial f_{\boldsymbol{p}}}{\partial \boldsymbol{p}}=g_{\boldsymbol{p}}-\frac{f_{\boldsymbol{p}}-\left\langle f_{\boldsymbol{p}}\right\rangle}{\tau},
$$

where $\boldsymbol{p}$ and $\boldsymbol{v}$ are the electron momentum and velocity, respectively, $g_{\boldsymbol{p}}$ is the generation rate of electrons in the state with the momentum $\boldsymbol{p}, \tau$ is the momentum relaxation time, and $\left\langle f_{\boldsymbol{p}}\right\rangle$ is the distribution function averaged over the momentum direction. Multiplying Eq. (3) by $e \boldsymbol{v}$ and summing up over $\boldsymbol{p}$ we obtain the equation for the photocurrent density $\boldsymbol{j}=\sum_{p} e \boldsymbol{v} f_{\boldsymbol{p}}$ in the absence of an in-plane bias

$$
-j \times \omega_{c}=G-\frac{j}{\tau},
$$

where $\boldsymbol{\omega}_{c}=\omega_{c} \hat{z}, \omega_{c}=e B_{z} / m_{\mathrm{CR}}$ is the cyclotron frequency, $\hat{z}$ is the unit vector pointing along the $z$ axis, and $\boldsymbol{G}=\sum_{p} e \boldsymbol{v} g_{p}$ is the rate of current generation.

The direction and magnitude of the vector $\boldsymbol{G}$ is determined by the asymmetry of scattering of surface carriers by static defects or phonons [see Eq. (2)] and the radiation absorption. In particular, the allowed asymmetric terms in Eq. (2) are determined by the spatial symmetry of the structure. For twodimensional systems on (013)-oriented surfaces, the scattering rate acquires asymmetric terms even for the magnetic field perpendicular to the surface. This is due to the absence of any nontrivial symmetry elements in the $\mathrm{C}_{1}$ point group describing such structures. Moreover, in this system, the scattering asymmetry in the surface plane is not forced by symmetry to certain crystallographic axes. Therefore, the photocurrent generation rate $\boldsymbol{G}$ in the out-of-plane magnetic field $B_{z}$ can be generally presented in the form

$$
\boldsymbol{G}=\boldsymbol{\gamma} \operatorname{I\eta }(\omega) B_{z},
$$

where $I$ is the radiation intensity, $\eta(\omega)$ is absorbance, and $\boldsymbol{\gamma}$ is the vector determined by the magnetic field induced asymmetry of electron scattering processes. As the direction of the vector $\gamma$ for the $C_{1}$ point group is not forced to a certain crystallographic axis by symmetry arguments, it may depend on temperature, gate voltage, radiation frequency, etc. Note that such a specific anisotropy of (013)-oriented structures has been demonstrated for photogalvanic currents excited by terahertz radiation in $\mathrm{HgTe}$ quantum wells at zero magnetic field [52]. For two perpendicular in-plane directions, the solution of Eq. (4) has the form

$$
\begin{aligned}
& j_{x}=\frac{\gamma_{x}+\omega_{c} \tau \gamma_{y}}{1+\left(\omega_{c} \tau\right)^{2}} \operatorname{I\eta }(\omega) B_{z}, \\
& j_{y}=\frac{\gamma_{y}-\omega_{c} \tau \gamma_{x}}{1+\left(\omega_{c} \tau\right)^{2}} \operatorname{I\eta }(\omega) B_{z} .
\end{aligned}
$$

Equations (6) show that the current (i) has a resonant behavior at $\omega=\omega_{c}$ caused by $\mathrm{CR}$ in the absorbance $\eta(\omega)$ and (ii) contains contributions even and odd in the magnetic field, both in agreement with experiment. The formation of photocurrent for positive and negative magnetic fields $B_{z}$ is illustrated in Fig. 10, which sketches the generation rate vector $\boldsymbol{G}$ for positive magnetic fields. The resulting photocurrent $\boldsymbol{j}$
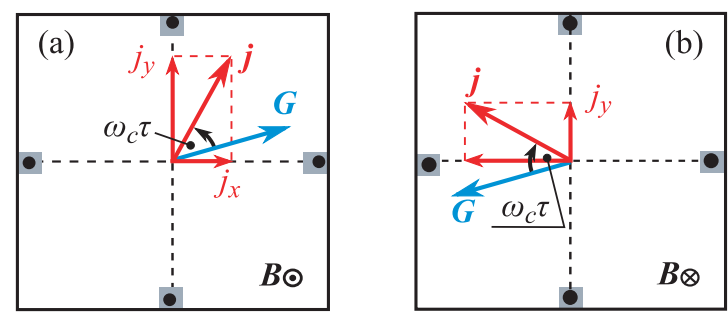

FIG. 10. (Color online) The vectors of photocurrent generation rate $\boldsymbol{G}$ and the steady-state photocurrents $\boldsymbol{j}$ for two opposite magnetic field directions [(a) and (b)]. The direction of photocurrent $j$ in the magnetic field is declined from the direction of $\boldsymbol{G}$ by the Hall angle $\arctan \left(\omega_{c} \tau\right)$. Figure illustrates that, for the given parameters, $j_{x}$ changes its sign while $j_{y}$ converses its sign when the magnetic field direction is reversed.

deviates from $\boldsymbol{G}$ due to the Hall effect. For opposite magnetic field, the vector $\boldsymbol{G}$ and the Hall rotation axis reverse as shown in Fig. 10(b). Consequently, the projections of the total photocurrent $\boldsymbol{j}$ onto $x$ and $y$ direction, $j_{x}$ and $j_{y}$, may be odd or even in magnetic field $\boldsymbol{B}$ as detected in experiment (see Fig. 9) and depicted in Fig. 10, but may also have the same parity [48]. In fact, the parity of the signal primarily depends on the photocurrent direction relative to the orientation of the contacts and the magnetic field strength determining the Lorentz force acting on moving carriers. Due to the fact that, as addressed above, the direction of the vector $\boldsymbol{G}$ is arbitrarily oriented for the $\mathrm{C}_{1}$ point group, the parity of the signal may change with temperature, gate voltage, radiation frequency, etc.

\section{SUMMARY}

To summarize, our results demonstrate that excitation of 80-nm-thick strained $\mathrm{HgTe}$ films by terahertz radiation results in cyclotron-resonance-induced photocurrents generated in the topologically protected surface states. The emergence of photocurrents reveals the importance of the magnetic field induced asymmetric scattering of nonequilibrium surface electrons. Due to the spin-momentum locking of surface electrons, the current is expected to be spin polarized; then, terahertz excitation results in a macroscopic surface spin polarization. As a specific feature of the (013)-oriented surface, this spin polarization has a nonvanishing out-of-plane component which can be probed by time-resolved Kerr rotation measurements. Our study demonstrates that the observed CRassisted photocurrent can be applied even for micrometer-size samples, e.g., gated structures, and for Fermi level lying in the conduction band, i.e., under conditions where transmission can hardly be measured.

\section{ACKNOWLEDGMENTS}

The authors thank E. G. Novik for discussions. The work was supported by DFG (Grant No. SPP 1666) and RFBR. K.-M.D., P.O., D.W., and S.D.G. acknowledge support from Elite Network of Bavaria (Grant No. K-NW-2013-247). G.V.B. and S.A.T. acknowledge support from RF President 
Grant No. MD-3098.2014.2, Program of the RAS, and the "Dynasty" Foundation.

\section{APPENDIX}

We calculate electron spectrum in low-symmetry strained $\mathrm{CdHgTe} / \mathrm{HgTe} / \mathrm{CdHgTe}$ structure on CdTe in the framework of six-band $\boldsymbol{k} \cdot \boldsymbol{p}$ model which is relevant for narrow-gap semiconductors. We consider the class of $(0 l h)$-oriented films ( $l$ and $h$ are integer numbers) which includes (011)-, (012)-, and (013)-oriented structures. In the basis of Bloch amplitudes of the $\Gamma_{6}$ and $\Gamma_{8}$ bands, the wave function has the form

$$
\Psi(\boldsymbol{\rho}, z)=\left(\begin{array}{l}
\psi_{\Gamma_{6},+1 / 2} \\
\psi_{\Gamma_{6},-1 / 2} \\
\psi_{\Gamma_{8},+3 / 2} \\
\psi_{\Gamma_{8},+1 / 2} \\
\psi_{\Gamma_{8},-1 / 2} \\
\psi_{\Gamma_{8},-3 / 2}
\end{array}\right) \exp \left(i \boldsymbol{k}_{\|} \cdot \boldsymbol{\rho}\right),
$$

where $\rho=(x, y)$ is the in-plane coordinate, $z$ is the growth axis, $\psi_{j}$ are the envelopes, $\boldsymbol{k}_{\|}=\left(k_{x}, k_{y}\right)$ is the in-plane wave vector.

The effective six-band Hamiltonian is given by

$$
H=\left(\begin{array}{ll}
H_{c c} & H_{c v} \\
H_{c v}^{\dagger} & H_{v v}
\end{array}\right)
$$

where the blocks $H_{c c}$ and $H_{v v}$ describe the conduction $\left(\Gamma_{6}\right)$ and valence $\left(\Gamma_{8}\right)$ intraband contributions, and the block $H_{c v}$ describes the band mixing. The block $H_{c c}$ is given by

$$
H_{c c}=I_{2 \times 2}\left[E_{c}(z)+\frac{\hbar^{2} \boldsymbol{k}[2 F(z)+1] \boldsymbol{k}}{2 m_{0}}+\Xi_{c} \operatorname{Tr} \epsilon\right],
$$

where $I_{2 \times 2}$ is the $2 \times 2$ identity matrix, $E_{c}(z)$ is the conduction band profile, $\boldsymbol{k}=\left(k_{x}, k_{y},-i \partial / \partial_{z}\right), F(z)$ is a parameter accounting for contribution from remote bands, $\Xi_{c}$ is the $\Gamma_{6}$-band deformation potential constant, and $\epsilon$ is the train tensor. The block $H_{c v}$ has the form

$$
H_{c v}=\left(\begin{array}{cccc}
-\frac{1}{\sqrt{2}} P k_{+} & \sqrt{\frac{2}{3}} P k_{z} & \frac{1}{\sqrt{6}} P k_{-} & 0 \\
0 & -\frac{1}{\sqrt{6}} P k_{+} & \sqrt{\frac{2}{3}} P k_{z} & \frac{1}{\sqrt{2}} P k_{+}
\end{array}\right),
$$

where $P$ is the Kane matrix element and $k_{ \pm}=k_{x} \pm i k_{y}$.

The block $H_{v v}$ is given by

$$
H_{v v}=E_{v}(\boldsymbol{r})+H_{L}^{(\mathrm{i})}+H_{L}^{(\mathrm{a})}+H_{\mathrm{BP}}^{(\mathrm{i})}+H_{\mathrm{BP}}^{(\mathrm{a})},
$$

where $E_{v}(\boldsymbol{r})$ is the valence band profile, $H_{L}^{(\mathrm{i})}, H_{L}^{(\mathrm{a})}, H_{\mathrm{BP}}^{(\mathrm{i})}$, and $H_{\mathrm{BP}}^{(\mathrm{a})}$ are the isotropic and anisotropic parts of the Luttinger and Bir-Pikus Hamiltonians,

$$
\begin{aligned}
& H_{L}^{(\mathrm{i})}=\frac{\hbar^{2}}{2 m_{0}}\left[-\boldsymbol{k}\left(\gamma_{1}+\frac{5}{2} \gamma_{2}\right) \boldsymbol{k}+2(\boldsymbol{J} \cdot \boldsymbol{k}) \gamma_{2}(\boldsymbol{J} \cdot \boldsymbol{k})\right], \\
& H_{\mathrm{BP}}^{(\mathrm{i})}=\left(a+\frac{5}{4} b\right) \operatorname{Tr} \epsilon-b \sum_{\alpha} J_{\alpha}^{2} \epsilon_{\alpha \alpha}-b \sum_{\alpha \neq \beta}\left\{J_{\alpha}, J_{\beta}\right\}_{s} \epsilon_{\alpha \beta},
\end{aligned}
$$

$\gamma_{1}, \gamma_{2}$, and $\gamma_{3}$ are contributions to the Luttinger parameters from remote bands, $\boldsymbol{J}$ is the vector composed of the matrices of the angular momentum $\frac{3}{2},\left\{J_{\alpha}, J_{\beta}\right\}_{s}=\left(J_{\alpha} J_{\beta}+J_{\beta} J_{\alpha}\right) / 2, a$, $b$, and $d$ are the $\Gamma_{8}$-band deformation potential constants. The explicit form of the terms $H_{L}^{(\mathrm{a})}$ and $H_{\mathrm{BP}}^{(\mathrm{a})}$ related to the cubic anisotropy of host crystals depends on the coordinate frame used.

In the coordinate frame relevant to $(0 l h)$-oriented structures $x\|[100], y\|[0 h \bar{l}]$, and $z \|[0 l h]$, the terms $H_{L}^{(\mathrm{a})}$ and $H_{\mathrm{BP}}^{(\mathrm{a})}$ assume the form

$$
\begin{aligned}
H_{L}^{(\mathrm{a})}= & \frac{2 \hbar^{2}}{m_{0}}\left\{\left\{J_{x} J_{y}\right\}_{s}\left(\gamma_{3}-\gamma_{2}\right) k_{x} k_{y}+\left\{J_{x} J_{z}\right\}_{s}\left\{\gamma_{3}-\gamma_{2}, k_{z}\right\}_{s} k_{x}\right. \\
& +\left[\left\{J_{y} J_{z}\right\}_{s} \cos 2 \theta+\frac{J_{z}^{2}-J_{y}^{2}}{2} \sin 2 \theta\right] \\
& \times\left[\left\{\left(\gamma_{3}-\gamma_{2}\right), k_{z}\right\}_{s} k_{y} \cos 2 \theta\right. \\
+ & \left.\left.\frac{k_{z}\left(\gamma_{3}-\gamma_{2}\right) k_{z}-\left(\gamma_{3}-\gamma_{2}\right) k_{y}^{2}}{2} \sin 2 \theta\right]\right\}, \\
H_{\mathrm{BP}}^{(\mathrm{a})}= & -2\left(\frac{d}{\sqrt{3}}-b\right)\left\{\left\{J_{x} J_{y}\right\}_{s} \epsilon_{x y}\right. \\
& +\left\{J_{x} J_{z}\right\}_{s} \epsilon_{x z}+\left[\left\{J_{y} J_{z}\right\}_{s} \cos 2 \theta+\frac{J_{z}^{2}-J_{y}^{2}}{2} \sin 2 \theta\right] \\
& \left.\times\left[\epsilon_{y z} \cos 2 \theta+\frac{\epsilon_{z z}-\epsilon_{y y}}{2} \sin 2 \theta\right]\right\},
\end{aligned}
$$

where $\theta=\arctan (l / h)$ is angle between the growth direction [0lh] and the [001] axis, $\theta \approx 18.4^{\circ}$ for (013)-oriented structures.

$\mathrm{HgTe}$ and $\mathrm{Cd}_{0.65} \mathrm{Hg}_{0.35}$ Te layers adopt the in-plane lattice structure of CdTe buffer which leads to the in-plane strain of the layers described by the strain tensor components $\epsilon_{x x}=\epsilon_{y y}=$ $a_{\mathrm{CdTe}} / a_{0}-1$, where $a_{\mathrm{CdTe}}$ is the lattice constant of CdTe and $a_{0}$ is the equilibrium lattice constant of the considered layer. Out-of-plane components of the strain tensor in each layer can be found by minimizing the elastic energy. Such calculations for $(0 l h)$-oriented structures yield

$$
\begin{aligned}
\epsilon_{z z} & =\frac{c_{11}^{2}+2 c_{11}\left(c_{12}-c_{44}\right)+c_{12}\left(-3 c_{12}+10 c_{44}\right)-\left(c_{11}+3 c_{12}\right)\left(c_{11}-c_{12}-2 c_{44}\right) \cos 4 \theta}{-c_{11}^{2}-6 c_{11} c_{44}+c_{12}\left(c_{12}+2 c_{44}\right)+\left(c_{11}+c_{12}\right)\left(c_{11}-c_{12}-2 c_{44}\right) \cos 4 \theta} \epsilon_{\|}, \\
\epsilon_{z y} & =\frac{\left(c_{11}+2 c_{12}\right)\left(c_{11}-c_{12}-2 c_{44}\right) \sin 4 \theta}{-c_{11}^{2}-6 c_{11} c_{44}+c_{12}\left(c_{12}+2 c_{44}\right)+\left(c_{11}+c_{12}\right)\left(c_{11}-c_{12}-2 c_{44}\right) \cos 4 \theta} \epsilon_{\|},
\end{aligned}
$$

$\epsilon_{x z}=0$, where $c_{11}, c_{12}$, and $c_{44}$ are elastic constants.

We have performed calculation of the electron spectrum and wave functions for the parameters listed in Table I.
The Schrödinger equation with the matrix Hamiltonian (A2) is solved numerically by expanding the envelope functions $\psi_{j}(z)$ in series of the harmonic oscillator functions 
TABLE I. Parameters of HgTe and CdTe.

\begin{tabular}{cccccc}
\hline \hline & $E_{g}(\mathrm{eV})$ & $\gamma_{1}$ & $\gamma_{2}$ & $\gamma_{3}$ & $2 m_{0}(P / \hbar)^{2}(\mathrm{eV})$ \\
\hline $\mathrm{HgTe}$ & $-0.303^{\mathrm{a}}$ & $4.1^{\mathrm{a}}$ & $0.5^{\mathrm{a}}$ & $1.3^{\mathrm{a}}$ & $18.8^{\mathrm{a}}$ \\
$\mathrm{CdTe}$ & $1.606^{\mathrm{a}}$ & $1.47^{\mathrm{a}}$ & $-0.28^{\mathrm{a}}$ & $0.03^{\mathrm{a}}$ & $18.8^{\mathrm{a}}$ \\
& $F$ & $a(\mathrm{eV})$ & $b(\mathrm{eV})$ & $d(\mathrm{eV})$ & $\Xi_{c}(\mathrm{eV})$ \\
\hline $\mathrm{HgTe}$ & $0^{\mathrm{a}}$ & $-0.13^{\mathrm{b}}$ & $-1.5^{\mathrm{d}}$ & $-8.0^{\mathrm{d}}$ & $-3.82^{\mathrm{f}}$ \\
$\mathrm{CdTe}$ & $-0.09^{\mathrm{a}}$ & $0.756^{\mathrm{c}}$ & $-1.0^{\mathrm{e}}$ & $-4.4^{\mathrm{e}}$ & $-2.687^{\mathrm{c}}$ \\
& $a_{0}(\AA)$ & $c_{11}(\mathrm{Mbar})$ & $c_{12}(\mathrm{Mbar})$ & $c_{44}(\mathrm{Mbar})$ & \\
$\mathrm{HgTe}$ & $6.46^{\mathrm{e}}$ & $0.597^{\mathrm{b}}$ & $0.415^{\mathrm{b}}$ & $0.226^{\mathrm{b}}$ & \\
$\mathrm{CdTe}$ & $6.48^{\mathrm{e}}$ & $0.562^{\mathrm{b}}$ & $0.394^{\mathrm{b}}$ & $0.206^{\mathrm{b}}$ & \\
\hline \hline
\end{tabular}

${ }^{a}$ Reference [46].

${ }^{\mathrm{b}}$ Reference [53].

${ }^{\mathrm{c}}$ Reference [54].

${ }^{\mathrm{d}}$ Reference [55].

${ }^{\mathrm{e}}$ Reference [56].

${ }^{\mathrm{f}}$ Reference [57].

following the procedure described for (001)-oriented structures in Ref. [46].

The calculated spectrum for the (013)-oriented structure is shown in Fig. 5. The wave functions of states emerging in the band gap of bulk strained HgTe are shown in Fig. 11. The states are localized at the film interfaces and predominantly formed from the heavy-hole states. Despite the fact that six-band $\boldsymbol{k} \cdot \boldsymbol{p}$ model used in the calculations neglects bulk inversion asymmetry of $\mathrm{HgTe}$ and the corresponding Dresselhaus spinorbit interaction, we have found that the spin carried by surface states in (013)-oriented structures for $k_{x} \neq 0$ has an out-ofplane component. The out-of-plane polarization defined by

$$
P_{z}=\frac{\int\left(\left|\psi_{\Gamma_{8},+3 / 2}\right|^{2}-\left|\psi_{\Gamma_{8},-3 / 2}\right|^{2}\right) d z}{\int\left(\left|\psi_{\Gamma_{8},+3 / 2}\right|^{2}+\left|\psi_{\Gamma_{8},-3 / 2}\right|^{2}\right) d z}
$$

is about 0.1 for the surface states presented in Fig. 11.

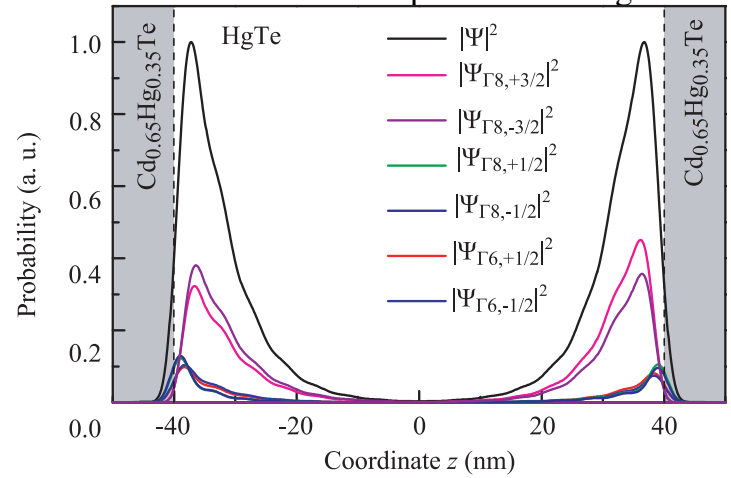

FIG. 11. (Color online) Wave functions of edge states in (013)grown 80-nm strained $\mathrm{HgTe}$ film. Calculations are carried out for the electron energy $E=11 \mathrm{meV}$, the wave vector components $k_{x}>0$ and $k_{y}=0$, and the built-in electric field $E_{z}=2 \mathrm{kV} / \mathrm{cm}$.

The emergence of the out-of-plane spin component is attributed to the cubic anisotropy incorporated in the Hamiltonian (A2) together with inversion symmetry breaking at surfaces. Indeed, the six-band $\boldsymbol{k} \cdot \boldsymbol{p}$ Hamiltonian of bulk crystal corresponds to the $O_{h}$ point group containing the spatial inversion. At a surface, the symmetry $z \leftrightarrow-z$ is broken which leads, for (013)-oriented surface, to the reduction of system symmetry to the $C_{s}$ point group containing the only nontrivial element $x \leftrightarrow-x$. Such a symmetry reduction enables the emergence of out-of-plane spin polarization for surface states with $k_{x} \neq 0$. We note that Dresselhaus spin-orbit interaction steaming from bulk inversion asymmetry of the host zinc-blende structure, which in not included in the model Hamiltonian, will lead to an out-of-plane spin polarization for surface states with $k_{y} \neq 0$.
[1] A. H. Castro Neto, F. Guinea, N. M. R. Peres, K. S. Novoselov, and A. K. Geim, Rev. Mod. Phys. 81, 109 (2009).

[2] K. S. Novoselov, V. I. Fal'ko, L. Colombo, P. R. Gellert, M. G. Schwab, and K. Kim, Nature (London) 490, 192 (2012).

[3] J. E. Moore, Nature (London) 464, 194 (2010).

[4] M. Z. Hasan and C. L. Kane, Rev. Mod. Phys. 82, 3045 (2010).

[5] X.-L. Qi and S.-C. Zhang, Rev. Mod. Phys. 83, 1057 (2011).

[6] M. M. Glazov and S. D. Ganichev, Phys. Rep. 535, 101 (2014).

[7] C. R. Becker, V. Latussek, A. Pfeuffer-Jeschke, G. Landwehr, and L. W. Molenkamp, Phys. Rev. B 62, 10353 (2000).

[8] C. R. Becker, V. Latussek, G. Landwehr, and L. W. Molenkamp, Phys. Rev. B 68, 035202 (2003).

[9] S. Dvoretsky, N. Mikhailov, Y. Sidorov, V. Shvets, S. Danilov, B. Wittman, and S. Ganichev, J. Electron. Mater. 39, 918 (2010).

[10] M. Baenninger, M. König, A. G. F. Garcia, M. Mühlbauer, C. Ames, P. Leubner, C. Brüne, H. Buhmann, L. W. Molenkamp, and D. Goldhaber-Gordon, J. Appl. Phys. 112, 103713 (2012).

[11] P. Ballet, C. Thomas, X. Baudry, C. Bouvier, O. Crauste, T. Meunier, G. Badano, M. Veillerot, J. P. Barnes, P. H. Jouneau, and L. P. Levy, J. Electron. Mater. 43, 2955 (2014).

[12] C. R. Becker, Phys. Status Solidi 251, 1125 (2014).
[13] B. A. Bernevig, T. L. Hughes, and S. C. Zhang, Science 314, 1757 (2006).

[14] M. S. König, S. Wiedmann, C. Brüne, A. Roth, H. Buhmann, L. Molenkamp, X. L. Qi, and S. C. Zhang, Science 318, 766 (2007).

[15] L. Fu, C. L. Kane, and E. J. Mele, Phys. Rev. Lett. 98, 106803 (2007).

[16] X. Dai, T. L. Hughes, X.-L. Qi, Z. Fang, and S.-C. Zhang, Phys. Rev. B 77, 125319 (2008).

[17] A. Roth, C. Brüne, H. Buhmann, L. W. Molenkamp, J. Maciejko, X.-L. Qi, and S.-C. Zhang, Science 325, 294 (2009).

[18] C. Brüne, C. X. Liu, E. G. Novik, E. M. Hankiewicz, H. Buhmann, Y. L. Chen, X. L. Qi, Z. X. Shen, S. C. Zhang, and L. W. Molenkamp, Phys. Rev. Lett. 106, 126803 (2011).

[19] J. N. Hancock, J. L. M. van Mechelen, A. B. Kuzmenko, D. van der Marel, C. Brüne, E. G. Novik, G. V. Astakhov, H. Buhmann, and L. W. Molenkamp, Phys. Rev. Lett. 107, 136803 (2011).

[20] K. C. Nowack, E. M. Spantor, M. Baenninger, M. König, J. R. Kirtley, B. Kalisky, C. Ames, P. Leubner, C. Brüne, H. Buhmann, L. W. Molenkamp, D. Goldhaber-Gordon, and K. A. Moler, Nat. Mater. 12, 787 (2013). 
[21] G. Grabecki, J. Wróbel, M. Czapkiewicz, Ł. Cywiński, S. Gierałtowska, E. Guziewicz, M. Zholudev, V. Gavrilenko, N. N. Mikhailov, S. A. Dvoretski, F. Teppe, W. Knap, and T. Dietl, Phys. Rev. B 88, 165309 (2013).

[22] J. B. Oostinga, L. Maier, P. Schuffelgen, D. Knott, C. Ames, C. Brüne, G. Tkachov, H. Buhmann, and L. W. Molenkamp, Phys. Rev. X 3, 021007 (2013).

[23] Z. D. Kvon, K.-M. Dantscher, C. Zoth, D. A. Kozlov, N. N. Mikhailov, S. A. Dvoretsky, and S. D. Ganichev, JETP Lett. 99, 290 (2014).

[24] D. A. Kozlov, Z. D. Kvon, E. B. Olshanetsky, N. N. Mikhailov, S. A. Dvoretsky, and D. Weiss, Phys. Rev. Lett. 112, 196801 (2014).

[25] C. Brüne, C. Thienel, M. Stuiber, J. Bottcher, H. Buhmann, E. G. Novik, C.-X. Liu, E. M. Hankiewicz, and L. W. Molenkamp, Phys. Rev. X 4, 041045 (2014).

[26] Q. Chen, M. Sanderson, J. C. Cao, and C. Zhang, Appl. Phys. Lett. 105, 202110 (2014).

[27] B. Büttner, C. X. Liu, G. Tkachov, E. G. Novik, C. Brüne, H. Buhmann, E. M. Hankiewicz, P. Recher, B. Trauzettel, S. C. Zhang, and L. W. Molenkamp, Nat. Phys. 7, 418 (2011).

[28] Z. D. Kvon, S. N. Danilov, D. A. Kozlov, C. Zoth, N. N. Mikhailov, S. A. Dvoretskii, and S. D. Ganichev, JETP Lett. 94, 816 (2011).

[29] M. Zholudev, F. Teppe, M. Orlita, C. Consejo, J. Torres, N. Dyakonova, M. Czapkiewicz, J. Wrobel, G. Grabecki, N. Mikhailov, S. Dvoretskii, A. Ikonnikov, K. Spirin, V. Aleshkin, V. Gavrilenko, and W. Knap, Phys. Rev. B 86, 205420 (2012).

[30] P. Olbrich, C. Zoth, P. Vierling, K.-M. Dantscher, G. V. Budkin, S. A. Tarasenko, V. V. Bel'kov, D. A. Kozlov, Z. D. Kvon, N. N. Mikhailov, S. A. Dvoretsky, and S. D. Ganichev, Phys. Rev. B 87, 235439 (2013).

[31] J. Ludwig, Yu. B. Vasilyev, N. N. Mikhailov, J. M. Poumirol, Z. Jiang, O. Vafek, and D. Smirnov, Phys. Rev. B 89, 241406(R) (2014).

[32] C. Zoth, P. Olbrich, P. Vierling, K.-M. Dantscher, V. V. Bel'kov, M. A. Semina, M. M. Glazov, L. E. Golub, D. A. Kozlov, Z. D. Kvon, N. N. Mikhailov, S. A. Dvoretsky, and S. D. Ganichev, Phys. Rev. B 90, 205415 (2014).

[33] M. Pakmehr, C. Bruene, H. Buhmann, L. W. Molenkamp, A. V. Stier, and B. D. McCombe, Phys. Rev. B 90, 235414 (2014).

[34] S. A. Tarasenko, M. V. Durnev, M. O. Nestoklon, E. L. Ivchenko, J.-W. Luo, and A. Zunger, Phys. Rev. B 91, 081302(R) (2015).

[35] M. Orlita, D. M. Basko, M. S. Zholudev, F. Teppe, W. Knap, V. I. Gavrilenko, N. N. Mikhailov, S. A. Dvoretskii, P. Neugebauer, C. Faugeras, A.-L. Barra, G. Martinez, and M. Potemski, Nat. Phys. 10, 233 (2014).

[36] J. G. Checkelsky, Y. S. Hor, M. H. Liu, D. X. Qu, R. J. Cava, and N. P. Ong, Phys. Rev. Lett. 103, 246601 (2009).

[37] A. A. Taskin and Y. Ando, Phys. Rev. B 80, 085303 (2009).

[38] J. G. Analytis, J. H. Chu, Y. Chen, F. Corredor, R. D. McDonald, Z. X. Shen, and I. R. Fisher, Phys. Rev. B 81, 205407 (2010).
[39] A. Shuvaev, G. V. Astakhov, C. Brüne, H. Buhmann, L. W. Molenkamp, and A. Pimenov, Semicond. Sci. Technol. 27, 124004 (2012).

[40] A. M. Shuvaev, G. V. Astakhov, G. Tkachov, C. Brüne, H Buhmann, L. W. Molenkamp, and A. Pimenov, Phys. Rev. B. 87, 121104(R) (2013).

[41] A. Shuvaev, A. Pimenov, G. V. Astakhov, M. Mühlbauer, C. Brüne, H. Buhmann, and L. W. Molenkamp, Appl. Phys. Lett. 102, 241902 (2013).

[42] J. Karch, C. Drexler, P. Olbrich, M. Fehrenbacher, M. Hirmer, M. M. Glazov, S. A. Tarasenko, E. L. Ivchenko, B. Birkner, J. Eroms, D. Weiss, R. Yakimova, S. Lara-Avila, S. Kubatkin, M. Ostler, T. Seyller, and S. D. Ganichev, Phys. Rev. Lett. 107, 276601 (2011).

[43] S. D. Ganichev, S. A. Tarasenko, V. V. Bel'kov, P. Olbrich, W. Eder, D. R. Yakovlev, V. Kolkovsky, W. Zaleszczyk, G. Karczewski, T. Wojtowicz, and D. Weiss, Phys. Rev. Lett. 102, 156602 (2009).

[44] S. D. Ganichev, Physica B (Amsterdam) 273-274, 737 (1999).

[45] E. Ziemann, S. D. Ganichev, I. N. Yassievich, V. I. Perel, and W. Prettl, J. Appl. Phys. 87, 3843 (2000).

[46] E. G. Novik, A. Pfeuffer-Jeschke, T. Jungwirth, V. Latussek, C. R. Becker, G. Landwehr, H. Buhmann, and L. W. Molenkamp, Phys. Rev. B 72, 035321 (2005).

[47] Note that the corresponding magnetic length $l_{B}=\sqrt{\hbar /(|e| B)}$ being about $16 \mathrm{~nm}$ for $B \sim 3.3 \mathrm{~T}$ is smaller than the thickness of $\mathrm{HgTe}$ films $(80 \mathrm{~nm})$, so that for the bulk carriers one would expect CR even for in-plane magnetic fields.

[48] Note that the difference in parity of these two resonant photocurrents is not the rule. Both resonant photocurrents may have opposite parity but may also have the same parity as detected in experiments on another sample.

[49] V. V. Bel'kov and S. D. Ganichev, Semicond. Sci. Technol. 23, 114003 (2008)

[50] V. V. Bel'kov and S. D. Ganichev, in Handbook of Spintronic Semiconductors, edited by W. M. Chen and I. A. Buyanova (Pan Stanford Publishing, Singapore, 2010), pp. 243-265.

[51] S. A. Tarasenko, Phys. Rev. B 77, 085328 (2008).

[52] B. Wittmann, S. N. Danilov, V. V. Bel'kov, S. A. Tarasenko, E. G. Novik, H. Buhmann, C. Brüne, L. W. Molenkamp, E. L. Ivchenko, Z. D. Kvon, N. N. Mikhailov, S. A. Dvoretsky, N. Q. Vinh, A. F. G. van der Meer, B. Murdin, and S. D. Ganichev, Semicond. Sci. Technol. 25, 095005 (2010).

[53] Chris G. Van de Walle, Phys. Rev. B 39, 1871 (1989).

[54] A. E. Merad, M. B. Kanoun, J. Cibert, H. Aourag, and G. Merad, Phys. Lett. A 315, 143 (2003).

[55] M. V. Yakunin, Fiz. Tekh. Poluprovodn. 22, 1452 (1988) [Sov. Phys.-Semicond. 22, 918 (1988)].

[56] S. Adachi, Handbook on Physical Properties of Semiconductors, Vol. 3 (Kluwer Academic Publishers, Berlin, 2004).

[57] V. Latussek, C. R. Becker, G. Landwehr, R. Bini, and L. Ulivi, Phys. Rev. B 71, 125305 (2005). 